\title{
Naoluo Xintong capsule ameliorates apoptosis induced by endoplasmic reticulum stress in rats with cerebral ischemia/ reperfusion injury
}

\author{
Sipeng Wu ${ }^{1,2}$, Xinxin Piao ${ }^{1,3,4}$, Ning Wang ${ }^{1,3,4}$, Yan Zhai ${ }^{1,3,4}$ \\ ${ }^{1}$ Key Laboratory of Chinese Medicinal Formula of Anhui Province, Anhui University of Chinese Medicine, Hefei, China; ${ }^{2}$ State Key Laboratory \\ of Cellular Stress Biology, School of Life Sciences, Xiamen University, Xiamen, China; ${ }^{3}$ Institute for Pharmacodynamics and Safety Evaluation of \\ Chinese Medicine, Anhui Academy of Chinese Medicine, Hefei, China; ${ }^{4}$ Key Laboratory of Xin'an Medicine, Ministry of Education, Hefei, China \\ Contributions: (I) Conception and design: S Wu, N Wang; (II) Administrative support: N Wang; (III) Provision of study materials or patients: N \\ Wang; (IV) Collection and assembly of data: S Wu, X Piao, Y Zhai; (V) Data analysis and interpretation: S Wu, N Wang; (VI) Manuscript writing: \\ All authors; (VII) Final approval of manuscript: All authors. \\ Correspondence to: Professor Ning Wang. Department of Pharmacy, Anhui University of Chinese Medicine, No. 1 Qian Jiang Road, Hefei, China. \\ Email: wnsci123@163.com.
}

Background: Naoluo Xintong (NLXT) capsuleis a newly developed drug recorded in the Chinese Pharmacopoeia. It is derived from traditional Chinese medicine (TCM) NLXT decoction, and has been widely used to treat cerebrovascular diseases in clinic. However, it is currently unknown whether it improve cerebral ischemia reperfusion (I/R) injury.

Methods: The effect of NLXT on regional cerebral blood flow (rCBF) was examined using Laser Doppler flower. The Terminal deoxyribonucleotide transferase-mediated Nick end labeling (Tunel) assay was performed to determine the effects of NLXT on apoptosis. Subsequently, cerebral water content and TTC staining were measured to assess cerebral edema and infarct volume, respectively. The protein expression levels were analyzed with Immunofluorescence and western blot assays.

Results: The results indicated that NLXT ameliorated MCAO-induced cerebral I/R injury by decreasing infract volume, inhibition of apoptosis, and upregulation rCBF. In addition, it decreased the expression of key protein involved endoplasmic reticulum (ER)-stress, including glucose-regulated protein 78 (GRP78), $\mathrm{C} / \mathrm{EBP}-$ homologous protein (CHOP) and Caspase-12 at $24 \mathrm{~h}$ following reperfusion. This was accompanied reduced degradation level of TRPC6 and increased phosphorylation of cAMP/Ca ${ }^{2+}$ response elementbinding protein (p-CREB), and decreased calpain-specific aII-spectrin breakdown product (SBDP145) activity. Interestingly, inhibition of mitogen-activated protein kinase (MEK) activity abolished the effect of NLXT on CREB activity.

Conclusions: Collectively, the results indicated that NLXT can improve I/R injury therapy by activating TRPC6/MEK/CREB signaling pathway to attenuate ER-stress related neuronal apoptosis.

Keywords: Naoluo Xintong capsule; cerebral ischemia reperfusion injury; endoplasmic reticulum stress; apoptosis

Submitted Feb 12, 2020. Accepted for publication Jul 20, 2020.

doi: $10.21037 /$ apm-20-387

View this article at: http://dx.doi.org/10.21037/apm-20-387 


\section{Introduction}

Stroke is characterized by high morbidity, mortality and disability rates, with World Health Organization (WHO) surveys showing that 15 million new stroke cases occur per year worldwide (1-3). Effective protection against ischemic brain damage is a worldwide conundrum, necessitating research into novel approaches for control of ischemiareperfusion (I/R) injury.

Cerebral I/R injury comprises various complex cascade reactions, such as calcium overload and neuron apoptosis. Key among them is the destruction of endoplasmic reticulum (ER) function, mediated by calcium overload, which can further induce ER-stress during ischemic (4). This process triggers expression of a series of associated factors or proteins, and initiates apoptosis, ultimately leading to neuronal death (5). Glucose-regulated protein 78 (GRP78), CHOP and caspase-12, comprise the key elements in the entire process of ER-stress. Generally, C/ EBP homologous protein (CHOP) inhibits expression of antiapoptotic protein Bcl-2 during ER-stress. On the other hand, direct activation of Caspase-12, an endoplasmic omentum protein, induces apoptosis only under the condition of ER-stress promotes its translocation to the cytoplasm, and eventually cleaves Caspase- 3 to cause apoptosis $(6,7)$. To date, research evidence has proven that regulating ER stress-related proteins inhibits apoptosis and exerts a remarkable protective effect on I/R injury (8). These studies further suggest that effectively controlling apoptosis-induced ER-stress might be a key approach for developing treatment therapies against ischemic stroke.

Transient receptor potential cation channels 6 (TRPC6) comprises a subfamily of non-selective calcium channels, which are widely expressed cell types, including neurons (9). Previous studies have shown that calpain activation leads to degradation of TRPC6 and neuronal damage in ischemia (10). Similarly, in vitro and in vivo studies have shown that CREB activation is a key downstream target for the neuronal protective effect of the TRPC6 channel (11). Consequently, it evident that inhibiting TRPC6 degradation may be a key therapeutic target for developing protection against ischemic brain injury.

To date, numerous basic experiments and clinic reports have reported the importance of traditional Chinese medicine (TCM) in conferring protection against cerebral I/R injury $(12,13)$. For example, Naoluo Xintong (NLXT) capsule, a modern hospital preparation created by Xin'an Medical master Letao Wang following years of clinical practice, at the First Affiliated Hospital of Anhui University of Chinese Medicine, has been found to increase drug concentration in the brain (Chinese national patent number: CN103182033A). Summarily, NLXT is prepared from six herbs namely, Astragalus membranaceus (Fisch.) Bge (Astragali Radix) 30 g, Ligusticum chuanxiong Hort. (Rhizoma Chuanxiong) $10 \mathrm{~g}$, Parmx notoginseng (Burk.) F. H. Chen (Notoginseng Radix Et Rhizome) 6 g, Gastrodia elata B1. (Gastrodiae Rhizoma) $10 \mathrm{~g}$, Angelica sinensis (Oliv.) Diels (Radix Angelicae Sinensis) 10 g, Carthamus tinctorius L. (Carthami Flos) $10 \mathrm{~g}$ and one animal medicine Scolopendra subspinipes mutilans L.Koch (Scolopendra) 4 g. A combination of 3D-UPLC-DAD and a mixture of standard compounds have enabled determination of fingerprints and eight active components in NLXT, as well as flavones, glucosides, polysaccharides and alkaloids, that are hypothesized to be the key biologically active components of the drug (14-17). Consequently, NLXT has been widely used to treat ischemic cerebrovascular diseases in clinics owing to its ability to enhance blood circulation and regulate meridians. Previous studies have demonstrated that NLXT remarkably improves survival rates and hemorheology in acute ischemic stroke patient (18-20). In addition, animal studies have affirmed NLXT's ability to control cerebral I/R injury in rats, owing to its $\mathrm{Ca}^{2+}$ content, ability to inhibit apoptosis, increase bFGF levels as well as regulate the coagulation and fibrinolytic system (21-26). However, to date, little is known regarding its specific antiapoptosis mechanisms. Given that ER stress-mediated neuronal apoptosis plays a key role in ischemic stroke, the present study sought to explore the underlying mechanism employed by NLXT to manage I/R injury, targeting the ER stress signaling pathway in rats induced by MCAO.

We present the following article in accordance with the ARRIVE reporting checklist (available at http://dx.doi. org/10.21037/apm-20-387).

\section{Methods}

\section{Animal}

Sprague-Dawley (SD) rats of SPF grade, weighing 240-280 g, were purchased from the Animal Experimental Center of Anhui Medical University and used in the present study. License No: SCXY (Anhui Province 2014-0009). All rats were allowed free access to standard diet and water. All animal handling and experimental procedures were approved by the ethics committee on Animal Care and 
Use of Anhui University of Chinese Medicine, China, and conducted in accordance with the guidelines provided by the National Institutes of Health on the Care and Use of Animals (NIH Publication number 80-23, revised 1986).

\section{Drug and reagents}

NLXT were prepared by the Department of Pharmacy, at the First Affiliated Hospital of Anhui University of Chinese Medicine (Batch number: 140701, 140802). Nimodipine tablets were purchased from Bayer Ltd., (batch No: 141207). Whereas PD98059 (MEK inhibitor) was acquired from Sigma-Aldrich Inc. DMSO was used at a concentration of $1.5 \mathrm{mg} / \mathrm{mL}$, and was prepared prior to application.

The TUNEL reagent kit was purchased from Roche (batch number: 10279600), BCA kit was from Beyotime (P0012), SDS-PAGE gel preparation kit from Biyotime (batch number: P0012A), and Nuclear/cytoplasmic protein extraction kit from Shanghai Yisheng Biotechnology Co., Ltd. Additionally, rat primary antibodies, against Caspase-12, CHOP, GRP78, Phospho-CREB, TRPC6, SBDP145, LaminB1, $\beta$-actin and horseradish peroxidase (HRP)-conjugated goat anti-rat IgG were purchased from Abcam (Cambridge, MA, USA), whereas Fluorescein isothiocyanate (FITC)-conjugated goat anti-rabbit IgG were purchased from Santa Cruz Biotechnology (Santa Cruz, CA, USA).

\section{Establishment of I/R model}

In vivo simulation of a cerebral I/R model was performed according the method described by Longa with minor modification (27). Summarily, rats were anesthetized with $4 \%$ isoflurane $(3 \mathrm{~mL} / \mathrm{kg}$ ) period, then their right sternocleidomastoid muscle separated from the sternoglossal muscle to expose the common carotid artery (CCA). Thereafter, the external carotid artery (ECA) was ligated by electrocauterization, and the CCA clamped. A small incision was then made, at the free end of the ECA, and a loose knot tied under the orifice. The prepared filament was inserted through the incision, along the ECA, then gently and slowly moved along the internal carotid artery (ICA), until there was slight resistance. Finally, an $18 \mathrm{~mm}$ embolus thread was inserted into the ICA, from the bifurcation of ICA and ECA, then the filament was removed to complete the reperfusion of the middle cerebral artery (MCA), after $2 \mathrm{~h}$ of occlusion. Rats in the sham group were subjected to a similar operation, but without inserting filament occlusion and reperfusion. Neurological deficits were evaluated using the Garcia JH score, whereas the neurological deficits and the regional cerebral blood flow (rCBF) was monitored by laser Doppler, during the operation.

\section{Grouping and dosages}

The whole experiment was divided into three batches. The first batch comprised 72 healthy male SD rats, randomly divided into six groups with 12 rats per group. There were the Sham group (S), Middle cerebral artery occlusion (MCAO) group (M), MCAO + positive drug group $(0.02 \mathrm{~g} / \mathrm{kg}$, Nimodipine, NM), MCAO + NLXT group [1.125 g/kg (N1), $2.25 \mathrm{~g} / \mathrm{kg}(\mathrm{N} 2), 4.50 \mathrm{~g} / \mathrm{kg}(\mathrm{N} 3)$, respectively]. NLXT $(1.125,2.25$ and $4.50 \mathrm{~g} / \mathrm{kg})$ and Nimodipine $(0.02 \mathrm{~g} / \mathrm{kg})$ doses were converted according to clinical adult dose. Rats in the MCAO + NLXT or MCAO + Positive drug groups had intragastrical administration NLXT or Nimodipine once a day, for 14 days following MCAO induction, and at onset of reperfusion. Rats in the Sham and MCAO groups had an intragastrical administration of normal saline.

In the second batch, 40 SD rats were randomly divided into four groups of 10 rats each. These were Sham, MCAO, MCAO + Positive drug $(0.02 \mathrm{~g} / \mathrm{kg}$, Nimodipine $0.02 \mathrm{~g} / \mathrm{kg})$ and MCAO + NLXT drug $(2.25 \mathrm{~g} / \mathrm{kg})$. After reperfusion, the MCAO + Positive drug and MCAO + NLXT drug groups were given their respective doses by intragastric administration, once a day for 7 days, whereas the Sham and MCAO groups were given normal saline for 7 days.

The third batch comprised 20 SD rats, randomly divided into five groups of 4 rats each. These included the Sham, MCAO, MCAO + NLXT drug $(2.25 \mathrm{~g} / \mathrm{kg}), \mathrm{MCAO}+\mathrm{MEK}$ inhibitor (PD98059) and MCAO + NLXT + PD98059 coadministration groups. After reperfusion, the MCAO + NLXT + PD98059 and MCAO + NLXT drugs were given their respective doses by intragastric administration of their respective dose, once a day for 7 days, whereas the Sham and MCAO groups were given physiological saline for 7 days. Rats were administered with PD98059, dissolved in $1 \%$ DMSO [final dose of $2.5 \mathrm{mg} / \mathrm{kg}(28)$ ], by caudal vein injection $30 \mathrm{~min}$ prior to $\mathrm{MCAO}$.

\section{Neurological impairment score}

Neurological function was evaluated using a blind method at 1, 7 and 14 after drug administration. Autonomic 
movement, symmetry of posture, extending function of forelimbs, and screen test were assessed using the Garcia JH score (29). In addition, the degree of neurological deficits was assessed based on six aspects, including bilateral tactile sensation and bilateral beard reflex. The highest score was 18 points, for a normal function, whereas the lowest score was 3 points for the most serious neurological deficits.

\section{Brain water content}

Rats were anesthetized with $4 \%$ isoflurane $(3 \mathrm{~mL} / \mathrm{kg})$ and their brain immediately removed, 30 minutes after the last drug administration. Residual blood, on the brain surface, was cleaned off with filter paper, and the wet brain mass determined using an electronic weight balance. Thereafter, the brain tissue was quickly dried in a dryer at a constant temperature of $115^{\circ} \mathrm{C}$, and the dry weight determined. Brain water content was calculated as follows; Brain water content $=($ wet weight - dry weight $) /$ wet weight $\times 100$.

\section{Assessment of cerebral blood flow}

Laser Doppler instrument was used to monitor $\mathrm{rCBF}$ at 7 and 14 days after ischemia and reperfusion process. The blood flow was monitored in infarcted area and its surrounding tissues. Rats were anesthetized and disinfected after per routine protocols. An incision was made on the skin, subcutaneous tissue along the midline and the right side of the skull. The fascia was separated to expose the skull. The location of $\mathrm{rCBF}$ was assessed in relation to Bregma: ML: $+2.0 \mathrm{~mm}, \mathrm{AP}:+1.0 \mathrm{~mm}$ (12). The infarct site in the cortex was considered as the ischemic peripheral area (the depth was within $1 \mathrm{~mm}$ of the cerebral cortex, that is, the ischemic penumbra area). The probe pedestal of the laser Doppler instrument was fixed with biological gum. Analysis and calibration were performed using routine debugging instrument.

\section{Terminal deoxyribonucleotide transferase-mediated Nick end labeling (Tunel)}

Brain sections were dewaxed and dehydrated as stated above, followed by blocking of the endogenous peroxidase. The sections were rinsed 3 times in PBS, and then incubated with the membrane breaking liquid for $10 \mathrm{~min}$ at room temperature. They were washed 3 times with PBS, and incubated with a mixture of reagent 1 and reagent 2 of the apoptosis detection kit (1:9) in a water bath with $37^{\circ} \mathrm{C}$ for 60 min. After washing with PBS, DAB color was developed. A brown precipitate was observed in the nucleus caused by the reaction between horseradish peroxidase and its substrate $\mathrm{DAB}$, indicating a positive TUNEL signal. Hematoxylin counterstaining was also performed, followed by dehydration, clearing, and sealing with tablets. The number of positive cells in three different visual fields on the ischemic side under a $400 \times$ objective were counted.

\section{TTC staining}

After 7 days of drug administration, the rats were anesthetized with $4 \%$ chloral hydrate, and decapitated immediately. The brain was immediately removed from the olfactory bulb to the cerebellum and placed on an ice bath platform. Coronal sections were cut from front to back, sectioned continuously into 5 slices. Brain slices were soaked in $4 \%$ TTC phosphate buffer solution at $37{ }^{\circ} \mathrm{C}$ for $10 \mathrm{~min}$. They were then fixed in $10 \%$ formalin solution for 24 hours. The brain slices were arranged in the order of anterior and posterior. Positive and negative sides of the brain slices were photographed by the pathological image collection and analysis system. Areas appearing red and white were measured, and the percentage of infarcted area was calculated with Image J software.

\section{Immunofluorescence}

Paraffin zed sections were dewaxed and dehydrated as described above. Sections were washed in PBS and placed in a box filled with EDTA solution (antigen retrieval buffer PH8.0) for antigen retrieval in a microwave. They were washed thrice with PBS and treat with BSA solution to block non-specific binding sites, followed by incubation in the primary antibody against TRPC6 at $4{ }^{\circ} \mathrm{C}$ overnight. Sections were washed and then incubated with secondary antibody in the fluorescein isothiocyanate (FITC) solution at room temperature for $50 \mathrm{~min}$. After washing with PBS, sections were dried slightly and sealed with an anti-fading tablet (Solarbio Technology Co., Ltd.). The nuclei were counterstained with 4',6-diamidino-2-phenylindole (DAPI). Finally, the sections were examined under a Nikon inverted fluorescence microscope and images were captured and saved.

\section{Western blot analysis}

Hippocampus tissues were homogenized with pre-cold 
RIPA lysis buffer and immediately mixed with $10 \mathrm{ul}$ of Phenylmethanesulfonyl fluoride (PMSF, Beyotime Institute of Biotechnology, Nanjing, China) and phosphatase inhibitor (1 mM) (Calbiochem, Merck, Germany), centrifuged at $12,000 \mathrm{rpm}$ at $4{ }^{\circ} \mathrm{C}$ for $15 \mathrm{~min}$. The concentration of the proteins was determined with the BCA assay. Protein were mixed with loading buffer and boiled for $10 \mathrm{~min}$ to denature the proteins. About $20 \mu \mathrm{g}$ of protein was loaded on the SDS-PAGE gel and separated by electrophoresis was completed. Proteins were transferred from the gel to a PVDF membrane by wet transfer method. The membrane was blocked using $5 \%$ skim milk powder for 2 hours, followed by incubation in the primary antibody (Caspase-12, 1:1,000; CHOP, 1:1,000; GRP78, 1:800; p-CREB, 1:500; TRPC6, 1:500; $\alpha$ II-spectrin, 1:500; $\beta$-actin, 1:1,000) overnight at $4{ }^{\circ} \mathrm{C}$. After washing with TBST, the membrane was incubated in the HRP conjugated secondary antibody (dilution: 1:10,000) for $2 \mathrm{~h}$ at room temperature. Finally, the membrane was emerged in the ECL reagent for 2 min to visualize the protein bands.

\section{Statistical analysis}

All data are expressed as mean \pm standard deviation $(x \pm s)$. Mean value between groups were compared with oneway analysis of variance (ANOVA) followed by Dunnett's test. All analyses were performed with SPSS 17.0 software package. $\mathrm{P}<0.05$ indicated a significant difference.

\section{Results}

\section{Effect of NLXT on neurological score, cerebral water content, cerebral blood flow (rCBF) and apoptosis}

The rCBF of the model group was significantly lower than that of the sham-operated group at time points ${ }^{*}, \mathrm{P}<0.05$; **, $\mathrm{P}<0.01$ ) (Figure 1A). Moreover, the medium dose of NLXT $(2.25 \mathrm{~g} / \mathrm{kg})$ significantly increased $\mathrm{rCBF}$ of the rats on day 7 and $14(\mathrm{P}<0.01)$. Interestingly, both low and high NLXT doses $(1.125$ and $4.5 \mathrm{~g} / \mathrm{kg}$ ) did not significantly increase $\mathrm{rCBF}$.

Cerebral I/R injury led to a significant deterioration in neurobehavioural score across days 1,7 and $14(\mathrm{P}<0.01)$, relative to the Sham group. However, both low and medium NLXT doses significantly restored neurological deficits, relative to the MCAO group $(\mathrm{P}<0.05, \mathrm{P}<0.01)$ on days 7 and 14. Subsequently, we found a significantly higher brain water content in the MCAO group than in the Sham group
$(\mathrm{P}<0.01)$. Conversely, both medium $(\mathrm{P}<0.05)$ and high $(\mathrm{P}<0.01)$ NLXT doses significantly reduced water content (Figure 1B,C).

Results from Tunel analysis revealed a significantly higher number of apoptotic cells in the hippocampus of MCAO rat relative to those in the Sham group $(\mathrm{P}<0.01)$. However, medium $(\mathrm{P}<0.05)$ and high $(\mathrm{P}<0.01)$ NLXT doses significantly reduced apoptosis of hippocampal neurons. Overall, these results indicate that NLXT mediates protection against cerebral I/R injury in rats (Figure 1D).

\section{Effect of NLXT on the volume of cerebral infraction after MCAO}

TTC staining is the key method for determining the success of the infarction model, therefore an important indicator during evaluation of a drug's protective effect. In the present study, we selected the $2.25 \mathrm{~g} / \mathrm{kg}$ for further evaluation of NXLT's effect on infraction volume. Consequently, brain slices of rat across each group were stained with TTC (Figure 2), photographed and the red and white areas measured. Determination of the percentage infarction areas, using Image J revealed that NLXT significantly reduced the white area compared to rats in the MCAO group $(\mathrm{P}<0.01)$.

\section{Effect of NLXT on ER stress-related proteins in MCAO- injured rats}

We analyzed expression profiles of ER stress-related marker proteins using western blot assay. Results revealed significantly higher $(\mathrm{P}<0.01)$ levels of $\mathrm{ER}$-stress marker caspase-12, GRP78, and CHOP in the model than the sham groups (Figure 3). Upon NLXT administration (2.25 g/kg), significantly lower levels of caspase-12, GRP78, and CHOP were observed in the model group. Taken together, these results indicate that NLXT protects against I/R injury thereby alleviating ER-stress induced cell apoptosis.

\section{Effect of NLXT on TRPC6/MEK/CREB pathways in MCAO-mediated rat}

During cerebral ischemia, Calpain is activated, then decomposed into a calpain-specific $\alpha \mathrm{II}$-spectrin protein product (SBDP145). Therefore, changes in patterns of SBDP145 protein expression can reflect the activity level of cerebral ischemia-reperfusion injury in rats. In the present study, we found a significant increase in the level of SBDP145 in rats with ischemia reperfusion $(\mathrm{P}<0.01)$ 

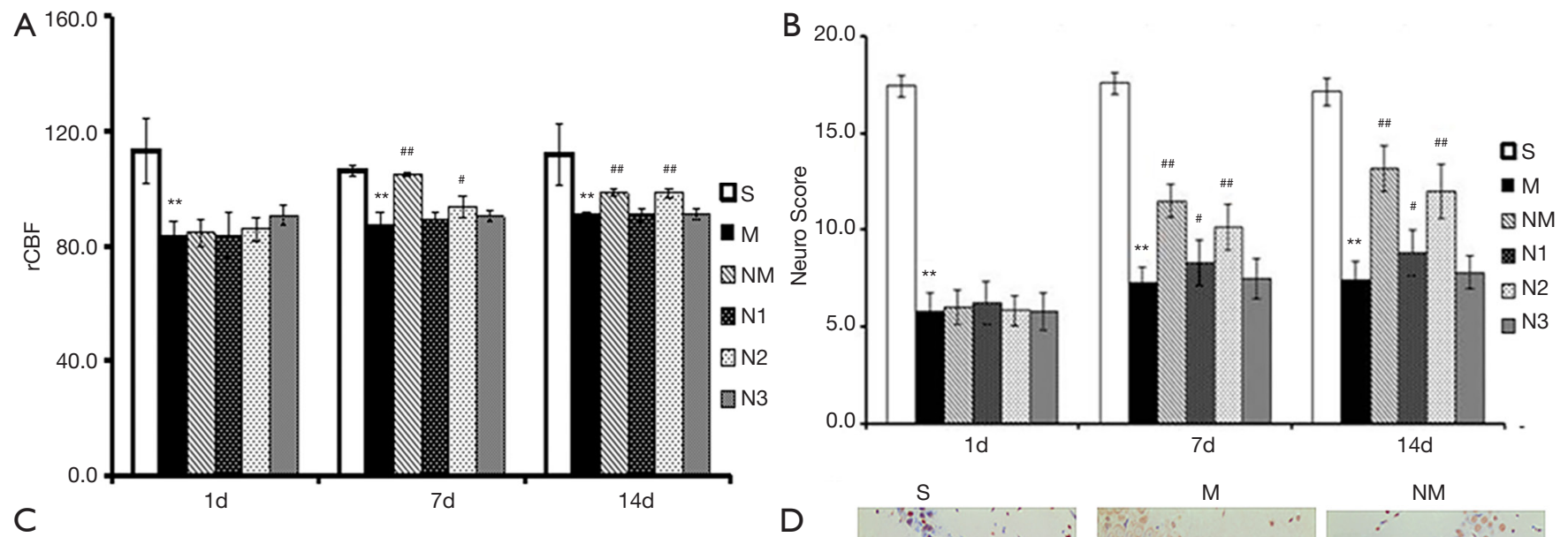

C
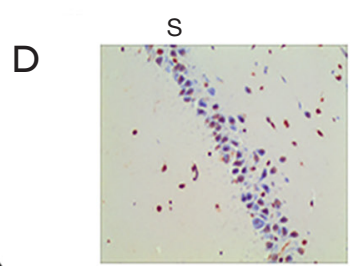

N1

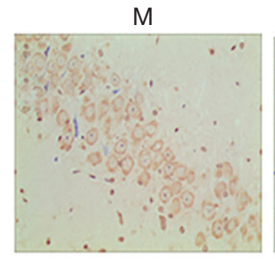

NM
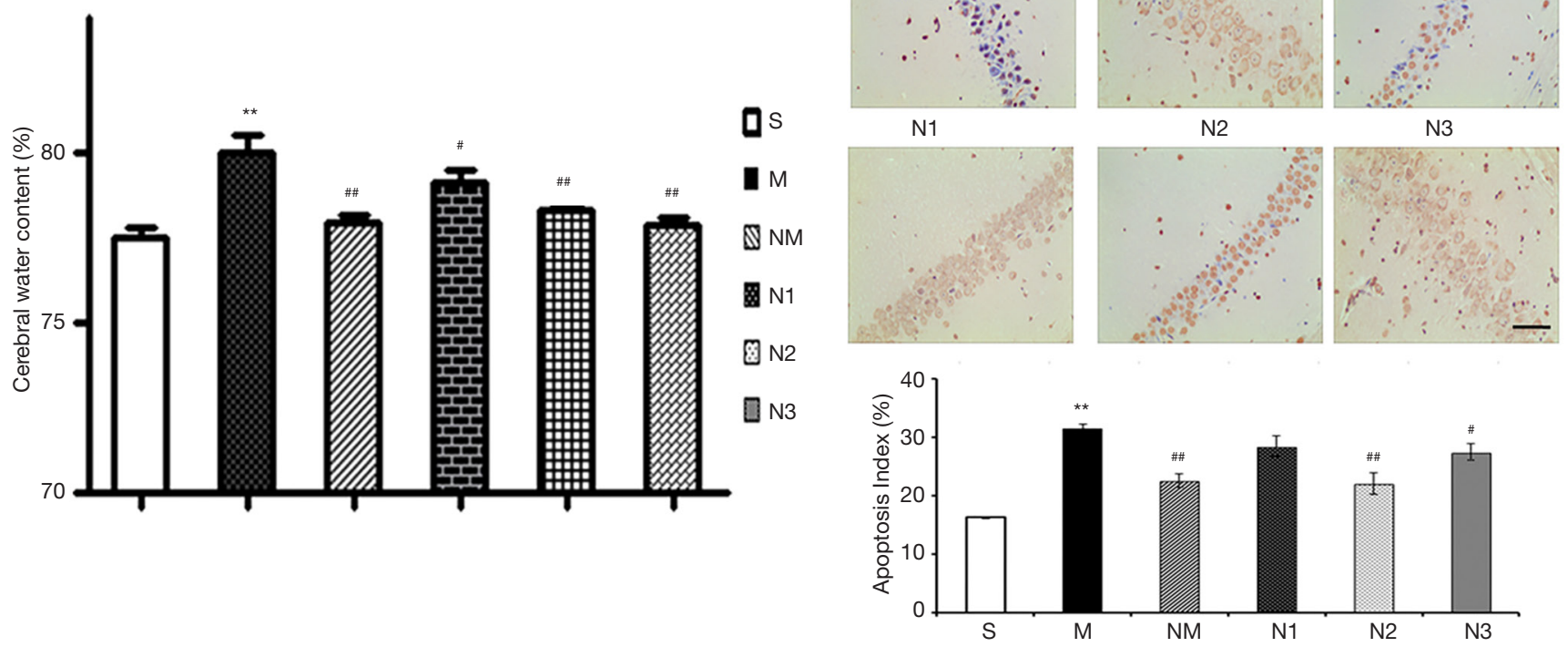

Figure 1 Effect of NLXT on rCBF, neuron score, cerebral water content and apoptosis in rats induced by MCAO. (A) The changes of rCBF in all group at 1, 7 and 14 days; (B) quantitative analysis of neurologic deficit scores in different groups at 1, 7 and 14 days; (C) quantitative analysis of cerebral water content at 14 days; (D) representative images of neuron stained with the terminal deoxynucleotidyl transferasemediated dUTP-fluorescein nick end labeling (TUNEL) (top) and Quantitative analysis of apoptosis (bottom). TUNEL-positive cells per field were counted. Scale bar $=50 \mu \mathrm{m}$ (×200 magnification). S: Sham group; M: MCAO group; NM: Nimodipine group; N1: NLXT (1.125 g/kg); $\mathrm{N} 2$ : NLXT $(2.25 \mathrm{~g} / \mathrm{kg})$ and N3: NLXT $(4.5 \mathrm{~g} / \mathrm{kg})$. Data were presented as mean $\pm \mathrm{SD} .{ }^{* *}, \mathrm{P}<0.01$ versus Sham group; ${ }^{\#} \mathrm{P}<0.05,{ }^{\# \#} \mathrm{P}<0.01$ versus MCAO group ( $\mathrm{n}=6)$. NLXT, Naoluo Xintong; rCBF, regional cerebral blood flow; MCAO, middle cerebral artery occlusion.

relative to those in the sham operation group (Figure $4 A$ ). In addition, NLXT significantly reduced SBDP145 expression $(\mathrm{P}<0.05)$, whereas significantly lower TRPC6 levels were observed in the model compared to the sham operation group $(\mathrm{P}<0.01)$. NLXT also significantly increased levels of TRPC6 in rats with $\mathrm{I} / \mathrm{R}$ injury $(\mathrm{P}<0.01)$. Similarly, images from immunofluorescence staining showed that NLXT improved TRPC6 changes in the hippocampal neuron membrane, consistent with western blots (Figure $4 B, C$ ). In addition, significantly lower $\mathrm{p}$-CREB levels were observed in the MCAO relative to the Sham group $(\mathrm{P}<0.01)$. However, NLXT administration led to a significantly increase in p-CREB expression. Coadministration of PD98059 reversed NLXT-mediated p-CREB up-regulation $(\mathrm{P}<0.05)$ (Figure $4 D)$. Overall, these results indicate that NLXT mediates protection 

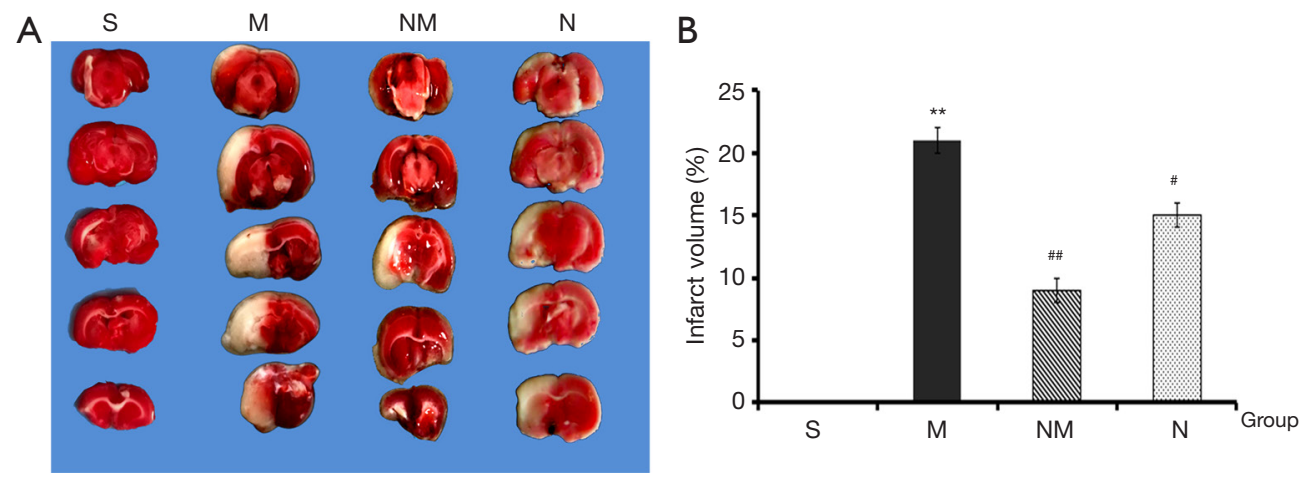

Figure 2 Effect of NLXT on infarct volume in rats induced by MCAO. Representative TTC-stained (left) brain slices and quantitative analysis (right) of infarct volume in all group. S: Sham group; M: MCAO group; NM: Nimodipine group and N: NLXT (2.25 g/kg), Data were presented as mean $\pm \mathrm{SD}$. **, $\mathrm{P}<0.01$ versus Sham group; ${ }^{\#} \mathrm{P}<0.05,{ }^{\# \#} \mathrm{P}<0.01$ versus MCAO group (n=6). NLXT, Naoluo Xintong; MCAO, middle cerebral artery occlusion.

A
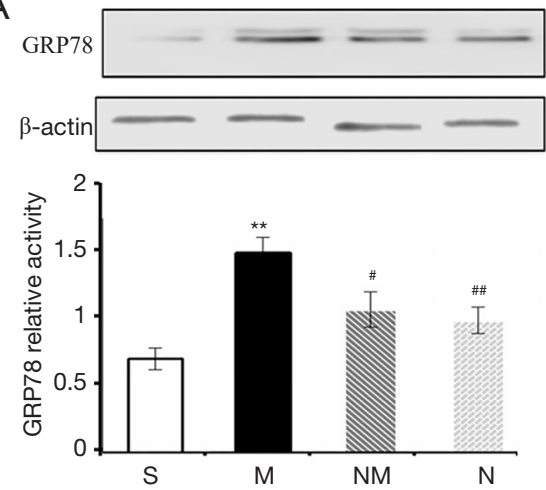

C
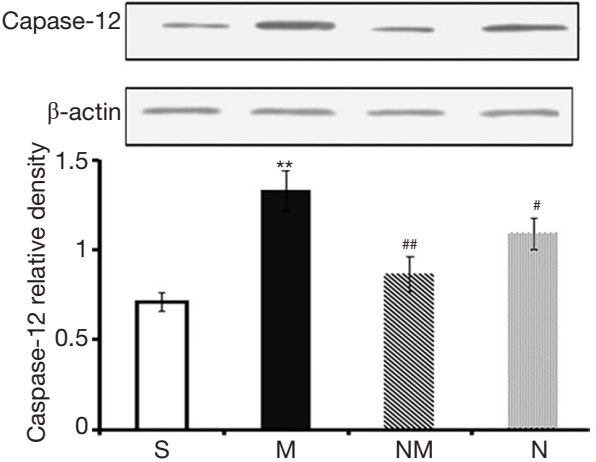

B
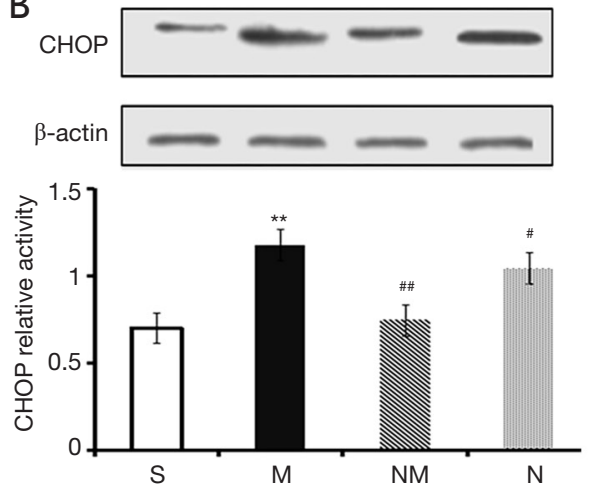

Figure 3 Effect of NLXT on ER stress-related proteins in MCAO-injured rat. Rats were subjected to 24 h of reperfusion, and NLXT $(2.25 \mathrm{~g} / \mathrm{kg})$ was given by intragastric administration for 7 days after reperfusion. Representative Western blot band (top) and the quantitative analysis (bottom) of the ratio of (A) GRP78, (B) CHOP and (C) caspase-12. S: Sham group; M: MCAO group; NM: Nimodipine group and N: NLXT $(2.25 \mathrm{~g} / \mathrm{kg})$. Data were presented as mean $\pm \mathrm{SD}$. ${ }^{* *}, \mathrm{P}<0.01$ versus Sham group; ${ }^{*} \mathrm{P}<0.05,{ }^{\# \#} \mathrm{P}<0.01$ versus MCAO group (n=3). NLXT, Naoluo Xintong; ER, endoplasmic reticulum; MCAO, middle cerebral artery occlusion. 

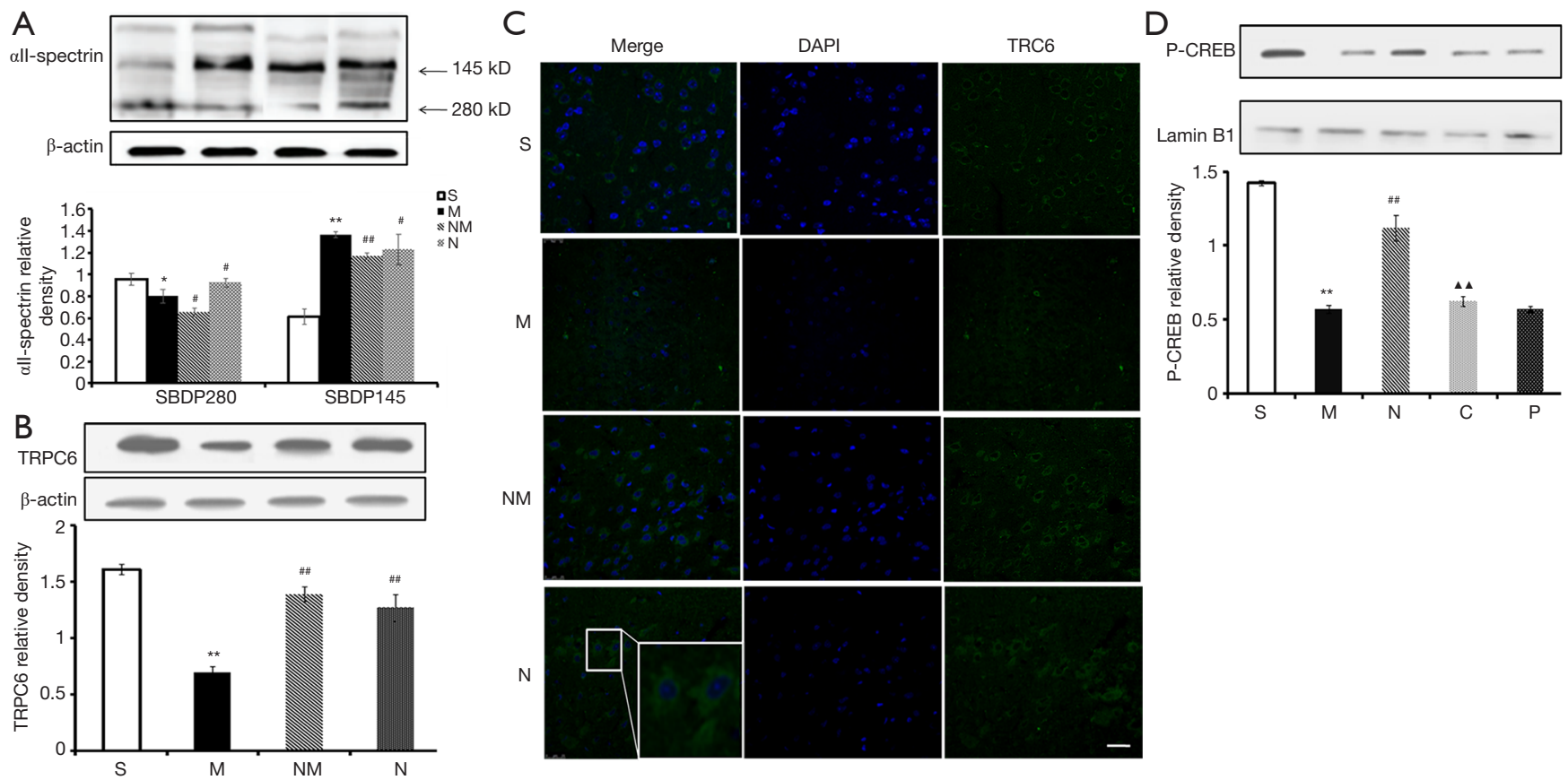

Figure 4 Effect of NLXT on TRPC6/MEK/CREB pathways in MCAO-injured rat. Rats were subjected to 24 h of reperfusion, and NLXT $(2.25 \mathrm{~g} / \mathrm{kg})$ was given by intragastric administration for 7 days after reperfusion. Representative Western blot band (top) and the quantitative analysis (bottom) of the ratio of (A) SBDP 145, (B) TRPC6 and (D) p-CREB. (C) Immunostaining labeled with TRPC6 (green) and co-staining with 4, 6-diamidino-2-phenylindole (blue) Scale bar =50 $\mu \mathrm{m}(\times 200$ magnification). S: Sham group; M: MCAO group; NM: Nimodipine group; N: NLXT (2.25 g/kg); C: combine group (NLXT + PD98059) and P: PD98059 group. Data were presented as mean \pm SD. *, $\mathrm{P}<0.05$; ${ }^{* *}, \mathrm{P}<0.01$ versus Sham group; ${ }^{*} \mathrm{P}<0.05$, and ${ }^{\# \#} \mathrm{P}<0.01$ versus $\mathrm{MCAO}$ group; ${ }^{\boldsymbol{\Lambda}}, \mathrm{P}<0.05$ versus NLXT (2.25 g/kg) group (n=3). NLXT, Naoluo Xintong; MCAO, middle cerebral artery occlusion.

against I/R injury by regulating the TRPC6/CREB pathway, in a MEK-dependent.

\section{Discussion}

In the present study, we have demonstrated NLXT's protective effect against I/R injury and elucidated its role on ER stress-induced neuronal apoptosis in MCAOinjured rats. Specifically, results from in vivo experiments demonstrated that NLXT treatment improves neurological defects and increases rCBF. Consequently, this effect reduces brain infarct size, and regulates expression of ER stress-related proteins in the brain of MCAO-treated rats.

Previous studies have implicated lack of energy supply to neuronal, following cerebral ischemic injury, in ineffective transport of $\mathrm{Ca}^{2+}$ from the cytoplasm. In fact, a release of calcium ions in the ER aggravates $\mathrm{Ca}^{2+}$ overload (30,31). Consequently, an influx of $\mathrm{Ca}^{2+}$ impels development of I/ $\mathrm{R}$ injury. Calpains are intracellular calcium-dependent cysteine endopeptidases that get activated by cytosolic $\mathrm{Ca}^{2+}$ overload. Numerous studies have demonstrated that activation of calcium activator calpain, after I/R injury, leads to degradation of $\alpha$ II-spectrin $(32,33)$ and TRPC6 $(34,35)$, which in turn increases extracellular calcium influx. In the present study, rats in the sham group exhibited significantly lower $\alpha \mathrm{II}$-spectrin protein decomposition product (SBDP145), whereas those in the NLXT group had high levels SBDP145 mediated by MCAO, but low calpain activity, as evidenced by low levels of calpain specific aIISBDP145.

Previous studies have shown that the TRPC subfamily is an essential channel in the regulation of calcium concentration, and plays a key role in the MCAO injury (36). In addition, TRPC6 promotes survival and differentiation of neurons induced by brain-derived nerve growth factor (BDNF) and has been directly associated with neuronal apoptosis in rats with cerebral ischemia $(36,37)$. Furthermore, numerous studies have shown that 
p-CREB not only effectively inhibits expansion of the infarction area, but also stimulates nerve re-generation $(38,39)$. These studies affirm that CREB activation is a critical target for neuroprotection from ischemic injury. However, appropriate concentrations of $\mathrm{Ca}^{2+}$ are needed to stimulate downstream CREB, through the TRPC6 channel, to promote neuronal survival (11). Therefore, modulating degradation of the TRPC6 channel maintains p-CREB, thereby exerting a remarkable protective effect on ischemic brain damage. In the present study, we investigated the effect of NLXT, and its underlying mechanisms, on TRPC6 signaling. Interestingly, immunofluorescence and SDS-PAGE results indicated that NLXT treatment mediated a significant reduction of MCAO-induced TRPC6 degradation. Particularly, significantly higher p-CREB levels were evident in the NLXT group, after ischemia, relative to the MCAO group. Conversely, significantly lower p-CREB levels were observed, following blocking of MEK activity, with a similar trend observed in either NLXT or coadministration (NLXT + PD98059) groups. Western blots demonstrated that NLXT, administered immediately following ischemia, stimulated the MEK signaling pathway and ultimately induced CREB activation. Overall, these findings indicated that NLXT blocks degradation of the calpain-mediated TRPC6 channel, which in turn activates CREB via the MEK signaling pathway.

Generally, UPR is a self-cytoprotective response that helps adaption, to re-establish ER homeostasis, although its continuous or prolonged activation can lead to cell death. In addition, an influx of calcium ions is an important signal for apoptosis, which also plays an important role in ER storage $(40,41)$. Generally, ER stress-induced apoptosis requires action of various related molecules, with previous evidence showing that an individual's GRP78 is directly correlated to the degree of ER-stress $(42,43)$. In addition, CHOP involvement in ER stress apoptotic pathway also represents a marker of ER stress. In fact, development of ER-stress results in an increase in CHOP, which subsequently inhibits anti-apoptosis by blocking Bcl-2expression. Similarly, Caspase-12 is present on ER's cytoplasmic surface, with previous studies showing that $\mathrm{Ca}^{2+}$ release activates procaspase-12 into Caspase-12, a special pathway for apoptosis induced by ER-stress $(44,45)$, and ER-stress mediated cell apoptosis plays a key role incidence of in stroke (46). In the present study, our results showed that NLXT intervention mediated a gradual decrease in I/R-induced neuron apoptosis in rats. In addition, electrophoretic bands revealed that ischemic reperfusion injury initiated ER-stress, which increased expression of GRP78, CHOP and Caspase-12. Conversely, NLXT intervention significantly reduced expression of these proteins. ER stress is a critical intracellular apoptosis signal. Results from Tunel staining indicated that NLXT administration mediated a reduction of apoptosis. Overall, these results suggested that NLXT plays a potential role in I/R mediated apoptosis linked with ER stress related pathway, providing useful insights for its use in treatment of cerebral I/R injury (Figure 5).

Taken together, our results confirmed that NLXT confers protection against cerebral I/R injury, by inhibiting ER-stress induced apoptosis and the TRPC6/MEK/CREB signal pathway. Nevertheless, the following further studies are needed to fully elucidate the complex mechanisms of ischemia-induced apoptosis; Firstly, it will be interesting to ascertain whether activated TRPC6 can activate CREB via the $\mathrm{CaM} / \mathrm{CaMKIV}$ signaling pathway and confer protection against I/R injury (47). It is also not known whether NLXT can modulate apoptosis through other pathways. Moreover, NLXT's effect on the dsRNA-activated protein kinase (PKR)-like ER kinase (PERK), inositol-Requiring Enzyme 1(IRE1) and Activating Transcription Factor 6 (ATF6) signaling pathways, during ER stress-induced apoptosis, remains unclear. Therefore, future studies are expected to assess whether NLXT may be beneficial in these pathways. Finally, further studies are expected to expound on the effect of various inhibitors, such as dantrolene and edaravone, which have been proven to be effective in attenuating ER stress after experimental cerebral ischemic injury (48). Future studies using ER-stress inhibitor are needed to enlarge our findings.

\section{Conclusions}

Summarily, our findings provide novel insights into NLXT's protective effects against I/R-induced brain injury. In addition, we have demonstrated the underlying mechanisms of NLXT action, including diminution of ER stressinduced neuronal apoptosis, inhibition of calpain proteolysis of TRPC6 as well as subsequent activation of CREB via the MEK signaling pathway. 


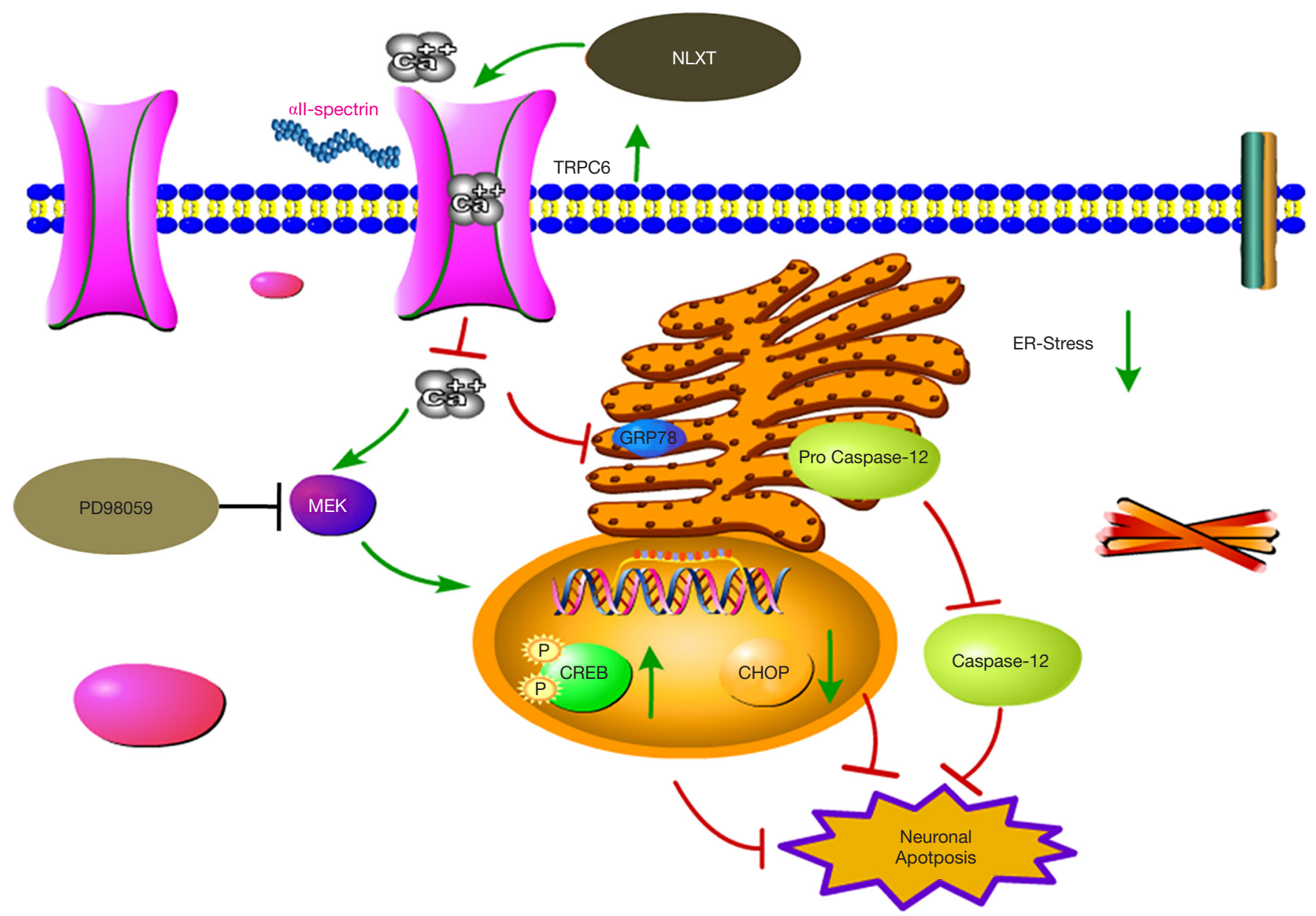

Figure 5 A schematic diagram illustrating the underlying molecular mechanism of NLXT on ER-stress induced apoptosis in rats with I/ $\mathrm{R}$ injury. NLXT mitigated I/R induced rats injury by suppressing over-activated ER-stress induced apoptosis, and such inhibition may be associated with the reduction of degradation of TRPC6, regulation of concentration of calcium. Finally, the activation of the TRPC6/MEK/ CREB signaling pathway involved in this protection process. NLXT, Naoluo Xintong; I/R, ischemia-reperfusion.

\section{Acknowledgments}

Funding: This work was supported by Academic assistance program for the top-notch innovative talents from universities in 2017 provided by Anhui Province Office of Education (gxbjZD15), the key projects of overseas visits of outstanding young backbone talents from universities in Anhui Province (gxfxZD2016117).

\section{Footnote}

Reporting Checklist: The authors have completed the ARRIVE reporting checklist. Available at http://dx.doi. org/10.21037/apm-20-387
Data Sharing Statement: Available at http://dx.doi. org/10.21037/apm-20-387

Conflicts of Interest: All authors have completed the ICMJE uniform disclosure form (available at http://dx.doi. org/10.21037/apm-20-387). The authors have no conflicts of interest to declare.

Ethical Statement: The authors are accountable for all aspects of the work in ensuring that questions related to the accuracy or integrity of any part of the work are appropriately investigated and resolved. All animal handling and experimental procedures were approved by the ethics committee on Animal Care and Use of Anhui University 
of Chinese Medicine, China, and conducted in accordance with the guidelines provided by the National Institutes of Health on the Care and Use of Animals (NIH Publication number 80-23, revised 1986).

Open Access Statement: This is an Open Access article distributed in accordance with the Creative Commons Attribution-NonCommercial-NoDerivs 4.0 International License (CC BY-NC-ND 4.0), which permits the noncommercial replication and distribution of the article with the strict proviso that no changes or edits are made and the original work is properly cited (including links to both the formal publication through the relevant DOI and the license). See: https://creativecommons.org/licenses/by-nc-nd/4.0/.

\section{References}

1. Beard JH, Ajiko MM, Lofgren J. From measuring disease burden to designing and evaluating solutionsglobal surgery research in evolution. JAMA Netw Open 2019;2:e186840.

2. Kim JS, Kim S, Lee SH, et al. Increased Risk of Ischemic Stroke during Sleep in Apneic Patients. J Clin Neurol 2018;14:174-8.

3. Xiong X, Naji DH, Wang B, et al. Significant Association between OPG/TNFRSF11B Variant and Common Complex Ischemic Stroke. J Stroke Cerebrovasc Dis 2018;27:1683-91.

4. Simard JC, Vallieres F, de Liz R, et al. Silver nanoparticles induce degradation of the endoplasmic reticulum stress sensor activating transcription factor-6 leading to activation of the NLRP-3 inflammasome. J Biol Chem 2015;290:5926.

5. Cybulsky AV. Endoplasmic reticulum stress, the unfolded protein response and autophagy in kidney diseases. Nat Rev Nephrol 2017;13:681-96.

6. Smith M, Wilkinson S. ER homeostasis and autophagy. Essays Biochem 2017;61:625-35.

7. Almanza A, Carlesso A, Chintha C, et al. Endoplasmic Reticulum Stress signalling - from basic mechanisms to clinical applications. FEBS J 2019;286:241-78.

8. Secondo A, Petrozziello T, Tedeschi V, et al. ORAI1/ STIM1 interaction intervenes in stroke and in neuroprotection induced by ischemic preconditioning through store-operated calcium entry. Stroke 2019;50:1240-9.

9. Montell C, Birnbaumer L, Flockerzi V. The TRP channels, a remarkably functional family. Cell 2002;108:595-8.
10. Du W, Huang J, Yao H, et al. Inhibition of TRPC6 degradation suppresses ischemic brain damage in rats. $\mathrm{J}$ Clin Invest 2010;120:3480-92.

11. Jia Y, Zhou J, Tai Y, et al. TRPC channels promote cerebellar granule neuron survival. Nat Neurosci 2007;10:559-67.

12. Wu SP, Li D, Wang N, et al. YiQi Tongluo Granule against Cerebral Ischemia/Reperfusion Injury in Rats by Freezing GluN2B and CaMK II through NMDAR/ ERK1/2 Signaling. Chem Pharm Bull (Tokyo) 2019;67:244-52.

13. She Y, Shao L, Zhang Y, et al. Neuroprotective effect of glycosides in Buyang Huanwu Decoction on pyroptosis following cerebral ischemia-reperfusion injury in rats. J Ethnopharmacol 2019;242:112051.

14. He L, Shi X, Seto SW, et al. Using 3D-UPLC-DAD and a new method-verification by adding mixture standard compounds to determine the fingerprint and eight active components of Naoluoxintong decoction. J Pharm Biomed Anal 2019;169:60-9.

15. Cheng G, Rong-Feng HU, Wang B, et al. Simultaneous Determination of Ferulic Acid and Gastrodin in Naoluoxintong Capsule by Dual-wavelength Highperformance Liquid Chromatography-Diode Array Detector. Journal of Anhui University of Chinese Medicine 2015;34;83-6.

16. Fang WY, Rong-Feng HU, Wang B, et al. Simultaneous Determination of Notoginsenoside and Ginsenosides in Compound Naoluoxintong Extracts by High-performance Liquid Chromatography. Journal of Anhui University of Chinese Medicine 2014;33;89-92.

17. Ding LZ, Rong-Feng HU, Wang J, et al. Quality control of Naoluo Xintong Capsules. Chinese Traditional Patent Medicine 2014;36;296-300.

18. Yang W, Xiang L, Jian W, et al. Clinical Observation on Naoluo Xintong Decoction for Treatment of Convalescent Cerebral Infarction Patients with Qi Deficiency and Blood Stasis Syndrome. Chinese Journal of Integrative Medicine on Cardio-/Cerebrovascular Disease 2013;12;1424-6.

19. Dai J, Zhou S, Ge Q, et al. Bi-directional regulation of cartilage metabolism by inhibiting BET proteins-analysis of the effect of I-BET151 on human chondrocytes and murine joints. J Orthop Surg Res 2018;13:118.

20. Chen C, Hu Q, Yan J, et al. Early inhibition of HIF-1alpha with small interfering RNA reduces ischemic-reperfused brain injury in rats. Neurobiol Dis 2009;33:509-17.

21. Wang J, Tan H, Jianpeng HU, et al. Effect of Naoluo 
Xintong Liquid on the Coagulation and Fibrinolytic System in Rats with Focal Cerebral Ischemia. J Tradit Chin Med 2012;53;1954-6.

22. Jian-Peng HU, Wang J, Wen-Ping WU, et al. Effect of naoluo xintong on expression of Fas, FasL in hippocampus CA1 area and Fas mRNA in the cortex of frontal or parietal lobe after local cerebral ischemia/reperfusion in MCAO rats. Chinese Journal of Pathophysiology 2007;23:720-4.

23. Wang J, Liu X. Effects of Naoluo Xintong Recipe on expression of HSP70 and bFGF in focal ischemiareperfusion rats. Journal of Chinese Integrative Medicine 2004;2:271-3.

24. Ling HE, Wen A, Yan Z, et al. Effects of Naoluo Xintong decoction on mRNA expressions of 5-hydroxytryptamine, dopamine, norepinephrine and 5-hydroxyindoleacetic acid in corpus striatum tissues of MCAO rats. Journal of Beijing University of Traditional Chinese Medicine 2015;38;740-4.

25. Wang L, Cao J, Zhu GQ, et al. Naoluoxintong reduces protein expression of TLR4,TRAF6 and TNF- $\alpha$ in frontal and parietal cortex of rats with focal cerebral ischemia reperfusion. Xi Bao Yu Fen Zi Mian Yi Xue Za Zhi 2018;34;702-7.

26. Henry RA, Schmit JA, Dieckman JF. The Analysis of Steroids and Derivatized Steroids by High Speed Liquid Chromatography. J Chromatogr Sci 1971;9:513-20.

27. Longa EZ, Weinstein PR, Carlson S, et al. Reversible middle cerebral artery occlusion without craniectomy in rats. Stroke 1989;20:84.

28. Yao C, Zhang J, Liu G, et al. Neuroprotection by (-)-epigallocatechin-3-gallate in a rat model of stroke is mediated through inhibition of endoplasmic reticulum stress. Mol Med Rep 2014;9:69-76.

29. Garcia JH, Wagner S, Liu KF, et al. Neurological deficit and extent of neuronal necrosis attributable to middle cerebral artery occlusion in rats. Statistical validation. Stroke 1995;26:627.

30. Mei Y, Thompson MD, Shiraishi Y, et al. Sarcoplasmic/ endoplasmic reticulum Ca 2 + ATPase C674 promotes ischemia- and hypoxia-induced angiogenesis via coordinated endothelial cell and macrophage function. J Mol Cell Cardiol 2014;76:275-82.

31. Ruiz A, Alberdi E, Matute C. Mitochondrial Division Inhibitor 1 (mdivi-1) Protects Neurons against Excitotoxicity through the Modulation of Mitochondrial Function and Intracellular Ca2+Signaling. Front Mol Neurosci 2018;11:3.
32. Bassett EA, Palanichamy K, Pearson M, et al. Calpastatin phosphorylation regulates radiation-induced calpain activity in glioblastoma. Oncotarget 2018;9:14597-607.

33. Takagi R, Ogasawara R, Takegaki J, et al. Past injurious exercise attenuates activation of primary calciumdependent injury pathways in skeletal muscle during subsequent exercise. Physiol Rep 2018;6:e13660.

34. Guo C, Ma Y, Ma S, et al. The role of TRPC6 in the neuroprotection of calycosin against cerebral ischemic injury. Sci Rep 2017;7:3039.

35. Zhang J, Mao X, Zhou T, et al. IL-17A contributes to brain ischemia reperfusion injury through calpain-TRPC6 pathway in mice. Neuroscience 2014;274:419-28.

36. Li H, Huang J, Du W, et al. TRPC6 inhibited NMDA receptor activities and protected neurons from ischemic excitotoxicity. J Neurochem 2012;123:1010-8.

37. Li Y, Jia YC, Cui K, et al. Essential role of TRPC channels in the guidance of nerve growth cones by brain-derived neurotrophic factor. Nature 2005;434:894-8.

38. Walton MR, Dragunow M. Is CREB a key to neuronal survival? Trends Neurosci 2000;23:48-53.

39. Finkbeiner S. CREB couples neurotrophin signals to survival messages. Neuron 2000;25:11-4.

40. Li L, Cui J, Liu Z, et al. Silver nanoparticles induce SH-SY5Y cell apoptosis via endoplasmic reticulumand mitochondrial pathways that lengthen endoplasmic reticulum-mitochondria contact sites and alter inositol-3-phosphate receptor function. Toxicol Lett 2018;285:156-67.

41. Ureshino RP, Rocha KK, Lopes GS, et al. Calcium signaling alterations, oxidative stress, and autophagy in aging. Antioxid Redox Signal 2014;21:123-37.

42. Yoshida H, Matsui T, Yamamoto A, et al. XBP1 mRNA Is Induced by ATF6 and Spliced by IRE1 in Response to ER Stress to Produce a Highly Active Transcription Factor. Cell 2001;107:881-91.

43. Groenendyk J, Sreenivasaiah PK, Kim DH, et al. Biology of endoplasmic reticulum stress in the heart. Circ Res 2010;107:1185-97.

44. Zinkstok SM, Vergouwen MDI, Engelter ST, et al. Safety and functional outcome of thrombolysis in dissectionrelated ischemic stroke: a meta-analysis of individual patient data. Stroke 2011;42:2515-20.

45. Graham HN: Green tea composition, consumption, and polyphenol chemistry. Prev Med 1992;21:334-50.

46. Zhu DY, Lau L, Liu SH, et al. Activation of cAMPresponse-element-binding protein (CREB) after focal cerebral ischemia stimulates neurogenesis in the adult 
dentate gyrus. Proc Natl Acad Sci U S A 2004;101:9453-7.

47. Lin Y, Chen F, Zhang J, et al. Neuroprotective effect of resveratrol on ischemia/reperfusion injury in rats through TRPC6/CREB pathways. J Mol Neurosci 2013;50:504-13.
48. Cao G, Zhou H, Jiang N, et al. YiQiFuMai powder injection ameliorates cerebral ischemia by inhibiting endoplasmic reticulum stress-mediated neuronal apoptosis. Oxid Med Cell Longev 2016;2016:5493279.
Cite this article as: Wu S, Piao X, Wang N, Zhai Y. Naoluo Xintong capsule ameliorates apoptosis induced by endoplasmic reticulum stress in rats with cerebral ischemia/reperfusion injury. Ann Palliat Med 2020;9(5):2913-2925. doi: 10.21037/apm-20387 